\title{
Production Planning and Control in Multi-Stage Assembly Systems: An Assessment of Kanban, MRP, OPT (DBR) and DDMRP by Simulation
}

Matthias Thürer* (corresponding author: matthiasthurer@workloadcontrol.com), Nuno O. Fernandes and Mark Stevenson

\author{
Name: $\quad$ Prof. Matthias Thürer \\ Institution: Jinan University \\ Address: $\quad$ School of Intelligent Systems Science and Engineering \\ Jinan University (Zhuhai Campus) \\ 519070, Zhuhai, PR China \\ E-mail: matthiasthurer@workloadcontrol.com \\ Name: $\quad$ Prof. Nuno O. Fernandes \\ Institution 1: Instituto Politécnico de Castelo Branco \\ Address 1: $\quad$ Av. do Empresário, 6000-767 \\ Castelo Branco - Portugal \\ Institution 2: ALGORITMI Research Unit (University of Minho) \\ Address 2: Campus de Gualtar \\ 4710-057 Braga \\ E-mail: $\quad$ nogf@ipcb.pt \\ Name: $\quad$ Prof. Mark Stevenson \\ Institution: Lancaster University \\ Address: $\quad$ Department of Management Science \\ Lancaster University Management School \\ Lancaster University \\ LA1 4YX - U.K. \\ E-mail: $\quad$ m.stevenson@lancaster.ac.uk
}

Keywords: Production Control; Kanban; MRP; Theory of Constraints; Demand Driven Material Requirements Planning. 


\title{
Production Planning and Control in Multi-Stage Assembly Systems: An Assessment of Kanban, MRP, OPT (DBR) and DDMRP by Simulation
}

\begin{abstract}
Multi-stage assembly systems where the demand for components depends on the market-driven demand for end products, are commonly encountered in practice. Production Planning and Control (PPC) systems for this production context include Kanban, Materials Requirement Planning (MRP), Optimized Production Technology (OPT), and Demand Driven MRP (DDMRP). All four of these PPC systems are widely applied in practice and literature abounds on each of these systems. Yet, studies comparing these systems are scarce and remain largely inconclusive. In response, this study uses simulation to assess the performance of all four PPC systems under different levels of bottleneck severity and due date tightness. Results show that MRP performs the worst, which can be explained by the enforcement of production start dates. Meanwhile, Kanban and DDMRP perform the best if there is no bottleneck. If there is a bottleneck then DDMRP and OPT perform the best, with DDMRP realizing lower inventory levels. If there is a severe bottleneck, then the performance results for DDMRP and OPT converge. This identification of contingency factors not only resolves some of the inconsistencies in the literature but also has important implications for the applicability of these four PPC systems in practice.
\end{abstract}

Keywords: Production Control; Kanban; MRP; Theory of Constraints; Demand Driven Material Requirements Planning. 


\section{Introduction}

This study uses simulation to assess the performance of different Production Planning and Control (PPC) systems in multi-stage assembly systems where the demand for components or product parts depends on the market-driven demand for end products. Our focus is hereby on a multi-item, multi-level production system that produces an unknown quantity of a fixed set of end products on a to-order basis. The objective is twofold: to provide guidance to companies regarding which PPC system to choose; and, to identify contingency factor that influence this decision. The study was triggered by observations in a major air-conditioner producer in Guangdong, PR China, which currently uses Material Requirements Planning (MRP; Orlicky, 1975, Vollmann et al., 1997), part of its Enterprise Resource Planning system, to control production. MRP is generally considered to be particularly suitable to this kind of production context, where component parts need to be coordinated for final assembly (Guide \& Srivastava, 2000). However, the firm struggled to control production without having to maintain high levels of in-process and finished goods inventories. It therefore decided to consider other alternatives suitable for its production context, such as Kanban (Sugimuri et al., 1977; Monden, 1983; Ohno, 1988), the Theory of Constraints' Optimized Production Technology solution (OPT; Goldratt \& Cox, 1984; Goldratt, 1990; Schragenheim \& Ronen, 1990; Simons Jr. \& Simpson III, 1997; Watson et al., 2007), and Demand Driven MRP (DDMRP; Ptak \& Smith, 2016; Miclo et al., 2019; Acosta et al., 2020). This study sets out to support this company and others in choosing between PPC alternatives, identifying contingency factors that likely influence implementation outcomes.

All of the above four PPC systems are widely applied in practice. For example, MRP continues to act as an important backbone of most PPC approaches in practice (Guide \& Srivastava, 2000; Hopp \& Spearman, 2011) since it can significantly improve the inventory and customer service levels of a production system (Whybark \& Williams, 1976; Ho, 1989). Meanwhile, White et al. (1999) reported that about 50\% of small and medium-sized manufacturers and around $70 \%$ of large manufacturers they surveyed have adopted some form of Kanban system. Similarly, White \& Prybutok (2001) reported that about $60 \%$ of nonrepetitive and $70 \%$ of repetitive manufacturers surveyed had adopted Kanban. Further, Mabin $\&$ Balderstone (2003) reviewed the literature on more than 80 successful implementations of the Theory of Constraints, with $80 \%$ reporting improvements in lead times and due date performance. Finally, Miclo et al. (2019) presented a list of companies that had successfully used DDMRP. 
Although all of the systems referred to above have been widely applied, literature comparing the different production planning and control systems remains scarce. Gupta \& Snyder (2009) identified only 20 articles comparing two or more of these systems and even found that most studies were inconclusive. Moreover, the available literature does not include the most recent advances in PPC systems, particularly DDMRP (Miclo et al., 2019), which has shown much early promise. In response, we use simulation to compare the performance of Kanban, MRP, OPT and DDMRP under different levels of bottleneck severity and due date tightness. Using different environmental factors and performance measures we seek to identify contingency factors that can be used to guide the choice of managers in practice, such as the managers of the company in our initial observations. Bottleneck severity was chosen since it influences complexity (Tenhiälä, 2011). Due date tightness was chosen given that realizing short customer lead times whilst maintaining high service rates is a key concern for the type of company under study.

The remainder of this study is structured as follows. Section 2 outlines the different PPC systems to be considered in this study. Section 3 then presents the simulation model used to evaluate performance, before the results are presented and discussed in Section 4. Finally, conclusions are summarized in Section 5, where managerial implications, limitations and future research directions are also outlined.

\section{Literature Review}

This section outlines the different PPC systems considered in this study. Section 2.1 introduces Kanban systems, Section 2.2 introduces MRP, Section 2.3 introduces OPT, and Section 2.4 introduces DDMRP. A discussion of the literature is then presented in Section 2.5, where we also outline the research question that motivated our study. Note that we do not aim to provide a comprehensive literature review of each system. There exists a broad literature on each of the different PPC systems, and providing a comprehensive review is beyond the scope of this study. For a literature review on Kanban, the reader is referred to, e.g. Lage Junior \& Godinho Filho (2010), for TOC to, e.g. Watson et al. (2007) or Ikeziri et al. (2019), while a review of the literature on DDMRP can be found in Miclo et al. (2019). Although there exist several reviews on the history of MRP and its origins (e.g. Jacobs \& Weston Jr, 2007; Wilson, 2016), no systematic review of the MRP literature could be identified. Finally, Lage Junior \& Godinho Filho (2010) identified more than 30 different Kanban systems. Clearly, including all of these systems in a comparison is beyond the scope of a single study. Thus, in our work, we attempt to stay as close as possible to the original description of a PPC system. 


\subsection{Kanban Systems}

There are several different Kanban systems presented in the literature. For a review and performance assessment, the reader is referred to Lage Junior \& Godinho Filho (2010) and Piplani \& Ang (2018), respectively. In this study, we focus on the use of Kanban as a replenishment system, as presented for example in Shingo (1989), since this is arguably the most commonly used version of Kanban in practice. This Kanban system uses inventory buffers, or so-called "supermarkets", that are replenished based on card signals. Kanban cards signal: (i) that parts have been consumed (and need to be replenished); and, (ii) which parts need to be replenished. Each part has an associated Kanban and there is no part without a Kanban (Ohno, 1988). The Kanban system described is inherently linked to a re-order point system (Shingo, 1989), as depicted in Figure 1. The main difference compared to a re-order point system is that: the number of Kanban cards represents the maximum inventory in the system ( $\mathrm{I}_{\min }+$ R.O.Q.); and there is a granularity in the measurement of the inventory level since it has to be expressed in terms of Kanban cards.

\section{[Take in Figure 1]}

A major issue with replenishment systems, such as the described Kanban system, is that inventory is always present. Kanban systems constantly seek to keep the inventory buffers at each stage of the manufacturing system full. While this ensures fast fulfillment of demand at downstream processes (including the end customer), it implies that there is inventory present even if there is no demand for parts or end products (Thürer et al., 2016). Moreover, product variability has to be limited since parts are produced in advance - otherwise, if there is a large number of different parts the inventory buffers become unmanageable (Suri, 1998).

\subsection{Material Requirements Planning (MRP)}

The development of MRP was triggered by the creation of unnecessary inventory in replenishment systems such as Kanban. Although MRP emerged in the late 1960s (Koh et al. 2002), it was only popularized after Orlicky (1975). While it is typically portrayed as being in contrast to just-in-time (Golhar \& Stamm, 1991; Gupta \& Snyder, 2009), MRP actually realizes the just-in-time principle by theoretically only producing parts when they are needed. In fact, MRP logic appears to preclude the use of any buffering mechanism (Guide \& Srivastava, 2000). It uses backward scheduling to calculate production start dates so that parts are available at the inventory points just when they are needed, instead of all the time (as in the above described Kanban system). Note that even if the MRP logic precludes inventory, safety stocks and safety 
lead times are often used to account for variability and nervousness (Whybark \& Williams 1976). There exist extensive publications concerning safety stock calculations for random demand of finished products (Louly \& Dolgui, 2013). Meanwhile the setting of the safety lead time is dependent on the quality of the lead time estimation, which should consider capacity and workloads (Rossi et al., 2017; Missbauer, 2020). In general, utilizing a time buffer results in better delivery performance when there is a variable supply, whereas having a safety stock results in improved delivery performance when there is unreliable demand information (van Kampen et al., 2010).

The MRP planning procedure relies on the basic tenet that there is both independent demand (the end product) and dependent demand (its parts or components), which are linked by a socalled bill-of-materials (BOM). Once the future demand for final products is known, MRP uses the BOM and a so-called lead time offset to backward schedule (or explode) the production requirements for each component. In this sense, MRP is not a replenishment policy that treats demand for all parts as being independent; rather, it is a scheduling procedure that creates a plan based on realized or forecasted future demand. The lead time offset is the equivalent to $t_{\text {rep }}$ in Figure 1. But instead of replenishing a fixed number of consumed parts, MRP produces a variable number of specific parts for future consumption.

\subsection{Optimized Production Technology (OPT) - Theory of Constraints}

When MRP emerged, computer power was a major issue (Jacobs \& Weston Jr, 2007). Moreover, the question remained regarding why all resources needed to be controlled, given that systems are typically constrained by a limited number of resources. In response, Eli Goldratt developed a production scheduling package called optimized production technology (OPT) in 1979 (Simons Jr. \& Simpson III, 1997). In contrast to MRP, where components are planned and released on a level-by-level basis (Steele et al., 2005), OPT schedules only the resource(s) that constrain(s) the system (later called the "Drum"). This drum schedule can be based on urgency or load-based considerations (Thürer \& Stevenson, 2018a). All nonconstraint resources are subordinated to the constraint schedule. OPT uses both a constraint buffer (i.e. the buffer before the bottleneck), to protect the bottleneck from starvation, and a shipping buffer (i.e. the finished goods buffer; see, e.g. Watson et al., 2007), to protect the customer. These buffers are either time (e.g. Radovilsky, 1998; Rahman, 1998; Schragenheim \& Ronen, 1990; Chakravorty \& Atwater, 2005; Golmohammadi, 2015) or a time-equivalent amount of work-in-process. 
For the simple case that there is a dominant flow and only one constraint, all parts downstream of the constraint are scheduled (similar to MRP), while all parts upstream of the constraint are replenished (similar to Kanban). This replenishment is triggered by the drum and communicated upstream by the so-called "Rope", which is the time offset between the scheduling of the drum and the release of raw materials (Ronen \& Starr, 1990). This offset is equivalent to $t_{\text {rep }}$ in Figure 1 and thus inherently linked to the buffer level. The rope ensures that the constraint buffers are kept small and stable and that jobs will pass through to the drum in the correct order. OPT is therefore now more commonly known as Drum-Buffer-Rope (DBR), a descriptor of the way in which it is realized (Simons Jr. \& Simpson III, 1997).

\subsection{Demand Driven MRP (DDMRP)}

The OPT approach puts a strong emphasis on resources and determining whether or not a particular resource is a constraint. But rather than taking such a resource-oriented approach, one could focus on the criticality of parts. In fact, it appears reasonable to retain critical parts in inventory to protect the system while permitting non-critical parts to be produced only when they are needed based on backward scheduling. This is the approach that is adopted by DDMRP, which was first presented as an appendix to the 3rd edition of Orlicky's Material Requirements Planning book in 2011 (see Ptak \& Smith, 2011) and later detailed by Ptak \& Smith (2016).

DDMRP keeps critical parts in inventory. If there are several critical parts in the production process then the system is decoupled by inventory buffers of these critical parts, as in the Kanban system described above. The main difference with DDMRP is that the Kanban system described above decouples all parts at all production stages, whereas DDMRP only decouples subsets of dependent parts (so called "pathways") in the product structure. The inventory buffers of critical parts are controlled by re-order points, which are calculated based on a socalled "net flow equation". As in MRP, time buckets (e.g. daily) are used, and for each bucket, the remaining inventory is calculated as the inventory on-hand plus the inventory on-order minus qualified demand. If the net flow position falls to a re-order point, a production order for the replenishment of critical parts is generated. The replenishment lead time for the re-order point calculation is based on the "decoupling lead time", which is defined as the longest, unprotected (unbuffered) lead-time in the BOM (Miclo et al., 2019) until the next decoupling point (or raw material). Once the production of critical parts is triggered, the production of noncritical parts (until the next decoupling point or raw material) is scheduled using MRP logic, which is referred to as a "decoupled explosion". 
In summary, DDMRP treats critical parts as independent and non-critical parts as dependent. It subdivides a system, where demand for parts depends on the market-driven demand for end products, into subsystems, where demand for non-critical parts depends on the demand for critical parts. The critical parts for which there are decoupling inventories are then controlled by a re-order point while non-critical parts are controlled using MRP (i.e. by backwards scheduling).

\subsection{Discussion of the Literature}

All four PPC systems discussed above are widely applied in practice in multi-stage assembly systems where the demand for components depends on the market-driven demand for end products, and literature abounds on each of these individual systems (see, e.g. Lage Junior \& Godinho Filho, 2010; Ikeziri et al., 2019). There are however few studies that compare the different systems, and those that are available are generally inconclusive in their findings (Gupta \& Snyder, 2009). There are two major studies that emerged after Gupta \& Snyder (2009); i.e. that were not included in their review: Jodlbauer \& Huber (2008) and Miclo et al. (2019). Our study differs from these two by identifying contingency factors that impact the applicability of the different PPC systems (and thus allow us to interpret the results of the studies discussed in Gupta \& Snyder (2009)). In contrast, Jodlbauer \& Huber (2008) focused on parameter sensitivity and Miclo et al (2019) on comparing more traditional PPC systems with DDMRP. Jodlbauer \& Huber (2008) compared the service levels of MRP, Kanban and OPT, concluding that MRP outperforms OPT and Kanban. This extends Steele et al. (2005) who compared MRP with OPT (or DBR) finding that OPT can outperform MRP, but that this depends on how MRP is implemented, i.e. how parameters are set. However, this finding is in contrast to Miclo et al. (2019) who compared MRP, Kanban and DDMRP and concluded that Kanban outperforms MRP (and that DDMRP overall performs the best). Hence, findings remain largely inconclusive even to-date.

It is argued here that the inconclusive nature of the findings is explained by the presence of contingency factors that determine the impact of a PPC system on performance. For example, in terms of factors external to the production control mechanisms, the existence and strength of bottlenecks has long since been shown to affect performance. Still, whether or not a bottleneck exists is not explicitly discussed in Jodlbauer \& Huber (2008) nor in Miclo et al (2019). Meanwhile, there is a clear bottleneck in Steele et al. (2005), but the impact of this factor is not assessed. Similarly, none of these studies considered the impact of due date tightness, although realizing short customer lead times while maintaining high service rates is 
a main concern of many companies in practice. This neglect of contingency factors is considered a major shortcoming, since these contingency factors determine the applicability of the different PPC systems to a given production context. As a consequence, managers are provided with very limited guidance in terms of how they should choose an appropriate system for their company. In response, this study asks:

What is the performance of Kanban, MRP, OPT and DDMRP in a multi-stage assembly system where the demand for components or product parts depends on the market-driven demand for end products, and how is this performance influenced by contingency factors?

Discrete event simulation is chosen to compare our four PPC systems and answer our research question. Kanban could be modelled by extending existing analytical models of basestock systems. However, the other three PPC systems use backward infinite loading to schedule production start dates. One approach to model these systems would be to extend Graves' (1986) Tactical Planning Model (TPM) as, for example, in Teo et al. $(2011,2012)$ for MRP. But the TPM is not easily extendable to OPT and DDMRP. We therefore argue that simulation is the best choice for our purpose of comparing the performance of the four PPC systems. We will use SIMIO software to build a generalized simulation model of a multi-stage assembly system where the demand for components or product parts depends on the market-driven demand for end products. To ensure verification, the simulation model can be requested from the corresponding author.

\section{Simulation Model}

The company that triggered our study produces 5 different categories of air conditioners, involving over 100 different products. The production process can slightly differ for the different categories and products and might be adapted to specific customer orders. Figure 2 shows the overall structure of the production process that consists of four different production lines - the mould injection, the condenser, the sheet metal and the control production - which feed into a central assembly line. The sheet metal production involves both the production process in the sheet metal plant as well as a subsequent painting process in a different plant and is therefore split in Figure 2. Each of the four production lines can be characterized as a disconnected flow line since production occurs on a limited number of routings through the process, while movement between the production steps is not fully automated so that inventory can build up between the steps. The same observation holds true for the final assembly operation. 
[Take in Figure 2]

Given the sheer size of the production system, we did not build a detailed model that accurately represents all aspects of the real system. Small systems provide a better insight into the role of operating variables and, in practice, large systems can often be decomposed into several smaller systems (Bokhorst et al. 2004). We therefore scanned the literature for a generalized model that matches this production system. This generalized model is based on Jodlbauer \& Huber (2008). However, there will be some modifications to allow for a fair comparison and the isolation of our two external contingency factors: bottleneck severity and due date tightness. First, we will use a shop with a single bottleneck, where non-bottleneck stations are balanced. This means that the processing times of parts are different when compared to Jodlbauer \& Huber (2008), where most stations had differing utilization rates. Second, the number of end products and part quantities will also be adjusted to create a symmetrical situation. Third, we will not consider set-up times, machine breakdowns or the scrap rate since we do not focus on robustness to stochastic factors (as in Jodlbauer \& Huber, 2008). To retain the capture of sources of variability, we will use stochastic rather than deterministic processing times.

\subsection{Job and Shop Characteristics}

The shop contains six stations, where each is a single, constant capacity resource. As in Jodlbauer \& Huber (2008), the shop has one bottleneck station - Station 3. There are eight different end products, with the structure of the products summarized in Table 1. For example, Product 1 needs two units of item B1 to be assembled at Station 1 (the final station). B1 is produced at Station 2 and requires three units of item C1. In total, each end product needs six assembly operations to be completed across stations 1 to 6 . Raw materials for Station 6 (the gateway station) are always available and there is an output buffer after each station.

\section{[Take in Table 1]}

Operation processing times before the adjustment to create the bottleneck follow a 2-Erlang distribution with a mean of 0.5 time units at stations 1 and 2 and 1.0 time units at the remaining four stations. Given the part quantities in Table 2, this results in a balanced shop. As in previous literature on bottleneck shops (e.g. Enns \& Prongue-Costa, 2002), non-bottlenecks are created by reducing the corresponding processing times. Three levels of bottleneck severity are considered: (i) no bottleneck, where processing times are not adjusted; (ii) moderate bottleneck, 
where processing times at all non-bottlenecks are reduced by 5\%; and, (iii) severe bottleneck, where processing times at all non-bottlenecks are reduced by $10 \%$. An equal adjustment was applied to all non-bottlenecks since the positioning of protective capacity is argued to have no effect on flow times (see Craighead et al., 2001).

The arrival of demand follows a stochastic process. The demand rate follows a Poisson distribution and all end products have the same probability of arrival. As in previous literature comparing these kinds of PPC systems (e.g. Steele et al., 2005; Jodlbauer \& Huber, 2008) there are fixed periods (e.g. weekly) in which demand occurs. In our study, the average demand arrival rate is 9 end products per period of 10 time units. The average demand has been set such that it deliberately results in a utilization level of $90 \%$ at the bottleneck station. Three settings for due date tightness are considered: tight, i.e. end products need to be delivered within 40 time units after the arrival date; medium, i.e. end products need to be delivered within 45 time units; and, loose, i.e. end products need to be delivered within 50 time units. These values are based on the realized average throughput times in preliminary simulation experiments.

\subsection{Production Planning and Control}

For all four PPC systems, demand is only satisfied at the due date. Finished products remain in a finished goods buffer until the due date. We acknowledge that at least part of the demand can be immediately fulfilled if there is an output buffer at the final station and that this may be an advantage. However, to create comparability across PPC systems, a due date (and thus a lead time allowance) is introduced to allow for a PPC system to plan and to execute the plan. Different parameter settings are considered for each PPC system since we cannot predict in advance which setting will lead to the best performance. To allow for a fair comparison a spectrum of the parameters was chosen for each PPC system such that we capture the best performance across the performance measures considered in this study. These parameter values were obtained through preliminary simulation experiments. The four systems are implemented as follows:

- Kanban: Products from Station 1's output buffer can immediately be moved into the finished goods inventory, which means demand is satisfied from this buffer (although the product is still not delivered to the customer prior to the due date). This frees up Kanban cards to move to the board of Station 1. Available parts for replenishment at the output buffer of Station 2 receive a Kanban from Station 1, detach the Kanban from Station 2 and move to Station 1 where they are processed. The Kanbans from Station 2 now trigger production at Station 3, and so on. The total number of Kanban cards, which defines the buffer size at each station, 
is tested at three levels and set to: 8,16 and 24 cards. The minimum buffer size is 8 since there must be a minimum inventory in the system for each product given that Kanban is a replenishment system. We consider Kanban cards to be part number-specific, with the total number of Kanbans being equally distributed over the number of part types.

- MRP: Production start dates are calculated for each part by backward scheduling using the lead-time offset. Production is then executed at each station according to the calculated start date (i.e. production cannot start if this start date is not reached). If the scheduled production start date of a station is reached, but not all parts are yet available from the upstream station, then and only then can safety stock be used. So, in contrast to a Kanban system where a given amount of inventory (the output buffer) is kept at a station to buffer demand, safety stock is a given amount of inventory that is kept at a station to buffer against a capacity shortage. As in Jodlbauer \& Huber (2008), we use safety stock for all parts (including the end products). The lead-time offset is given by the cumulative moving average, i.e. the average of all operation throughput times realized until the current simulation time plus a time buffer which is set to 0,2 and 4 time units. The safety stock level or buffer size at each stage is set to 0,8 and 16 items. Again, the total stock level is equally distributed over the number of part types.

- $O P T$ (or DBR): We use two buffers - an output buffer at Station 1 and an output buffer at Station 4. As in a Kanban system, both buffer against demand variation. Products from Station 1's output buffer can be moved into the finished goods inventory immediately, which means demand is satisfied from this buffer (although the product is still not delivered to the customer before the due date). Release dates are then calculated for each part at Station 3 (our bottleneck) by backward scheduling using the same lead time offset as for MRP. This creates the drum schedule. There is an output buffer at Station 4 protecting the bottleneck. The rope is calculated, again using the same lead time offset as for MRP. Identical buffer sizes are considered at both the finished goods buffer and at Station 4, and tested at three levels, namely with 16, 24 and 32 items. Again, the total buffer level is equally distributed over the number of part types.

- DDMRP: The first decision is the positioning of the buffer inventory. While there exists a vast literature on bottleneck detection (e.g. Roser \& Nakano, 2015), the matter of how to explore criticality is less explored. In general, the DDMRP literature buffers the vast majority of parts, e.g. 14 out of 16 in Miclo et al. (2019). To create a fair comparison with the other approaches, in this study we buffer all parts needed at Station 3 (the bottleneck) 
identically to OPT. To create completely decoupled sub-systems we also use an output buffer at Station 1. Products from Station 1's output buffer can immediately be moved into the finished goods inventory, which means demand is satisfied from this buffer (although the product is still not delivered to the customer before the due date). If the remaining inventory in the output buffer at Station 1 falls to a re-order point level, then a production order for the replenishment of critical parts is generated. This replenishment consumes parts that are buffered at the output buffer of Station 4. If the remaining inventory at this buffer falls to its re-order point level, then a production order for producing these critical parts from raw materials is generated. We use a min-max inventory policy (Lee \& Rim, 2019) with three levels for the re-order point (the Min): 1, 2 and 3 items. As for Kanban, one is the minimum level required. We use a continuous review policy, i.e. production orders are placed as soon as the inventory drops to or below the re-order point. Meanwhile, identical buffer sizes (the Max) are considered at both the output buffer at Station 1 and Station 4, and tested at three levels, namely 24, 32 and 40 items. Again, the total buffer level is equally distributed over the number of part types.

\subsection{Dispatching}

Each PPC system is typically evaluated using a different dispatching rule. Kanban is typically implemented with First Come First Served dispatching, MRP typically uses production start dates, as does OPT, while DDMRP uses the calculated net-requirements for prioritizing orders. However, there is no particular reason to restrict the combination of dispatching rule and PPC system. For example, dispatching based on net-requirements has also been used with Kanban systems (e.g. Ardalan \& Diaz, 2012). Since our focus is not on the dispatching rule itself, and we want to reduce its impact, we chose to use only one simple dispatching rule to prioritize order on the shop floor in combination with all four PPC systems: production start dates. The production start dates are calculated for each part by backward scheduling from the due date using a lead-time offset, which is given by the cumulative moving average, i.e. the average of all operation throughput times realized until the current simulation time.

\subsection{Experimental Design and Performance Measures}

Our study considers two external contingency factors: (i) three levels of the severity of the bottleneck (no bottleneck, moderate bottleneck, and severe bottleneck); and, (ii) three levels of due date tightness (tight, medium, and loose). For each PPC system, we used a full factorial design considering these two factors and the specific parameter settings. Each scenario of the 
experimental design was replicated 100 times. Results were collected over 13,000 time units following a warm-up period of 3,000 time units. These simulation conditions allow us to obtain stable results while keeping the simulation run time to a reasonable level.

As in previous literature comparing these PPC systems (e.g. Steele et al., 2005; Jodlbauer \& Huber, 2008; Miclo et al., 2019), three main performance measures are considered: the Service Level (SL), referring to the fraction of the number of customer orders delivered on time; the Finished Goods Inventory (FGI), which is the number of end products completed and assigned to a customer order; and the Work-In-Process (WIP) inventory, which is the number of parts (and end products) in the production system that departed from raw materials but did not yet enter into the FGI.

\section{Results}

This section first assesses the performance of each PPC system in isolation before a discussion is provided in Section 4.5. Here, we compare the different systems and reassess the PPC system used in the company that triggered this study.

\subsection{Performance Assessment: Kanban}

The performance results obtained for Kanban are summarized in Table 2. The following can be observed from the results:

- Bottleneck Severity: If there is a bottleneck, then the overall system utilization reduces, which leads to the expected improvement in performance observed.

- Due date tightness: This environmental factor has a negligible effect on performance. The due date tightness does not impact realized operation throughput times while the total throughput time is in general lower than the due date allowance.

- Parameter Setting: As expected, FGI and WIP measures increase with the inventory buffer size. At the same time, service levels are close to $100 \%$ for most settings. The only exception is an inventory buffer of 8 , if there is no bottleneck. Note that a lower inventory buffer was not possible given that we need at least 1 Kanban card for each of the eight end products.

[Take in Table 2]

\subsection{Performance Assessment: MRP}

The performance results obtained for MRP are summarized in Table 3. The following can be observed from the results: 
- Bottleneck Severity: If there is a bottleneck then overall system utilization reduces, which leads to the expected improvement in performance observed for all settings, except the setting where the time buffer and inventory buffer are zero. Here, service levels actually deteriorate. At non-bottleneck stations, realized operation throughput times get shorter, which leads to later releases of jobs to the system. This in turn reduces WIP levels, but at the cost of a deterioration in service level performance. This negative effect disappears if a time buffer or an inventory is used.

- Due date tightness: This environmental factor has a negligible effect on the service level under most settings. This comes somewhat as a surprise given that MRP backward schedules from the due date. Since production start dates are enforced, i.e. production cannot start before the production start date is reached, the system can only fulfil demand during the lead time allowance, i.e. the due date minus the calculated release date at the first station in the routing. This lead time allowance is not affected by due date tightness and simply given by the sum of the lead time offsets. The main effect is that slightly longer operation throughput times are realized for loose due dates (given our dispatching rule), which leads to longer lead time offsets. This in turn leads to earlier releases to the system and higher WIP levels.

- Parameter Setting: As expected, FGI and WIP measures increase with an increased inventory buffer size. Meanwhile, an increase in the time buffer leads to lower WIP levels and higher FGI levels since jobs are released earlier to the system. Both buffers have a positive impact on service level performance. However, an inventory buffer should be used to ensure high service levels in our modelled environment. Even when using a time buffer of 4 for each operation, the service level barely reached 50\% on-time delivery. Meanwhile, using an inventory buffer of 16 items without a time buffer yields a service level of more than $90 \%$. This aligns with van Kampen et al. (2010) who found that a safety stock results in a higher delivery performance compared to a time buffer where there is unreliable demand information. Another issue is that a time buffer equivalent to the inventory buffer (i.e. the time it needs to produce an equivalent amount of inventory) would require much larger planning periods and thus customer lead times.

[Take in Table 3]

\subsection{Performance Assessment: OPT (DBR)}

The performance results obtained for OPT are summarized in Table 4. The following can be observed from the results: 
- Bottleneck Severity: There is a very strong improvement in performance if there is a bottleneck. This improvement is likely to be higher than the improvement expected from the reduction in the system utilization, which can be explained by the PPC system's clear emphasis on the bottleneck constraint.

- Due date tightness: This environmental factor has a negligible effect on performance. The system can only fulfil demand during the lead time allowance, which is not affected by due date tightness. Meanwhile, any effect on the drum schedule caused by the realized operation throughput times is accommodated by the inventory buffer.

- Parameter Setting: As expected, FGI and WIP measures increase with the inventory buffer. Meanwhile, an increase in the time buffer leads to lower WIP levels and higher FGI levels since jobs are released earlier to the system. Both buffers have a positive impact on service level performance; however, as for MRP an inventory buffer should be used to ensure high service levels.

[Take in Table 4]

\subsection{Performance Assessment: DDMRP}

The performance results obtained for DDMRP are summarized in Table 5. The following can be observed from the results:

- Bottleneck Severity: If there is a bottleneck, then we can observe the expected improvement in performance.

- Due date tightness: This environmental factor has a negligible effect on the service level under most settings. The due date tightness does not impact realized operation throughput times. If the total throughput time is shorter than the lead time allowance, as for a re-order point of 1 , then the service level performance deteriorates at tighter due dates.

- Parameter Setting: As expected, FGI and WIP measures increase with the inventory buffer and re-order point. Increasing both the inventory buffer and re-order point has a positive impact on service level performance; however, the re-order point has the strongest effect. The re-order point should be set in such a way as to allow for the replenishment time. If the re-order point is too low then prolonged stock-outs may occur and the service level will suffer.

[Take in Table 5] 


\subsection{Discussion of Results}

\subsubsection{Performance Comparison}

Using Thompson (1967) as a theoretical lens, we can observe that the above systems differ in terms of the type of co-ordination mechanism applied. The Kanban system (as described above) controls production by mutual adjustment, i.e. responding to a deviation in the inventory level by attempting to stabilize this level through continuous replenishment. Meanwhile, MRP controls production via planned production start dates, i.e. MRP uses backward scheduling to produce parts only when they are needed whilst seeking to minimize the safety stock to account for variability and MRP nervousness. Finally, OPT and DDMRP use a combination of both approaches, as summarized in Table 6.

\section{[Take in Table 6]}

Our results re-emphasize that some form of mutual adjustment is required if there is variability and market-driven demand resulting, e.g. from make-to-order production. MRP performs far worse than the other three PPC systems tested in our study. Good service level performance can only be achieved by MRP with high inventories. The biggest issue however is not so much that production is planned. In fact, if the planning period is short enough, some form of mutual adjustment is realized by MRP, leading to improved performance (Steele et al., 2005). The major issue in our study is that no further adjustment to the plan is possible. In fact, the enforcement of production start dates, i.e. only starting processing at a station if the start date at this station has been reached, may often lead to worse performance than using the production start date as a priority measure, as for a dispatching rule (Thürer et al. 2019). In the latter case, variability in realized operation throughput times is levelled across stations. A major shortcoming of MRP systems in high-variety contexts is the assumption that individual production orders can be combined, as in repetitive contexts, and that predetermined lead-time offsets can be used when determining production start dates (Thürer et al., 2020). In highvariety contexts, the lead-time offset should consider the current workload situation (Teo et al., 2012; Missbauer, 2020) for each individual job. If this is not possible then the production start dates should allow for mutual adjustment.

The enforcement of production start dates in our study explains the discrepancy in results when compared to Jodlbauer \& Huber (2008), where MRP outperformed Kanban systems. Jodlbauer \& Huber (2008) did not enforce production start dates at stations, rather they applied a dispatching rule. Our study thus extends Jodlbauer \& Huber (2008) by arguing that it is specifically the enforcement of the production start dates that causes the worse performance of 
MRP. Meanwhile, our results align with Miclo et al. (2019), where MRP is clearly outperformed by Kanban and DDMRP. It is also this effect that partly explains the better performance observed for OPT and DDMRP for the scenarios in our study that do not have a bottleneck constraint. Both OPT and DDMRP also have a planning component but restrict enforced production start dates to a limited set of stations.

If there is a bottleneck then both OPT and DDMRP clearly outperform all other PPC systems considered in our study, since they realize high service levels at lower WIP and FGI levels than Kanban. It is apparent that there is no need for an inventory buffer if there is a capacity buffer. So, Kanban creates 'obvious' inventory waste (Hopp \& Spearman, 2004). However, the additional inventory is only obvious waste if the bottleneck is also obvious as in our study. As soon as there are shifting bottlenecks, Kanban may outperform PPC systems that only focus on the bottleneck, such as OPT and DDMRP, since the bottleneck may shift out of the control sphere of the PPC system (Thürer \& Stevenson, 2018b). Kanban leads to similar performance to OPT and DDMRP but without this risk. Meanwhile, DDMRP performs better than OPT, i.e. it realizes higher service levels at lower FGI and WIP levels, if there is a moderate bottleneck. This is mainly due to the use of a re-order point, which leads to lower average levels in the inventory buffer. DDMRP and OPT converge and become equivalent if there is a severe bottleneck. In fact, if criticality of a part is defined by the risk that the bottleneck starves then DDMRP and OPT become quite similar.

Finally, a summary of the applicability of the different PPC systems resulting from our discussion is provided in Table 7.

[Take in Table 7]

\subsubsection{Reassessment of the Triggering Case}

After the simulations and the performance comparison we reassessed the PPC system used in the company that triggered our study: the major air-conditioner producer in Guangdong, PR China. This company uses MRP, which is part of its Enterprise Resource Planning system. Yet, while it struggles with inventory, its performance appears to be much better than it should be, based on our simulation results. After closer analysis we observed that the company is not in fact using MRP per se. Rather, it is using some form of OPT or DDMRP system, informed by the calculations provided by the MRP system. Which perspective is taken, OPT or DDMRP, depends on whether or not final assembly is perceived to be the bottleneck - something we could not fully assess. In fact, the company has mainly two inventory buffers: a finished goods inventory, to protect the customer; and a deliberate parts buffer (of one day), to protect the final 
assembly line. This final assembly line has a fixed schedule and acts as the 'Drum' for OPT or provides the input for DDMRP's decoupled explosion. The production start dates for upstream stations are only used for priority dispatching. This reassessment highlights that all four PPC systems use similar elements: inventory and time buffers. They differ in terms of which element is used, where it is used, and what emphasis is given to each element. This study has focused on well-established PPC systems while future research could deconstruct these systems into their underlying elements, reconstruct new systems, or assess the contingency factors for each element.

\section{Conclusion}

Multi-stage assembly systems where the demand for components or product parts depends on the market-driven demand for end products, are commonly encountered in practice. Arguably the most commonly used PPC systems to control the flow of materials in this production context include Kanban, MRP, OPT, and DDMRP. But although all four PPC systems are widely applied in practice and there is a large body of literature on these systems, studies comparing these different systems are scarce and remain largely inconclusive. In response, this study asked: What is the performance of Kanban, MRP, OPT and DDMRP in a multi-stage assembly system where the demand for components or product parts depends on the marketdriven demand for end products, and how is this performance influenced by contingency factors? Simulation results were first analyzed for each PPC system in isolation before a comparison of all four PPC systems was provided. The results support our argument that there are indeed contingency factors that determine the impact of a PPC system. For example, the existence of a bottleneck, its severity and the degree of bottleneck shiftiness all have a strong impact on the choice of PPC system to implement. Meanwhile, our study has highlighted that due date tightness has a largely negligible impact on PPC system performance, i.e. due date tightness has only a minor or even no impact on realized operation throughput times. This does not however mean that due date tightness does not impact applicability - tighter due dates require PPC systems that realize shorter operation throughput times. These findings not only resolve some of the inconsistencies in the literature but also have important implications for the applicability of PPC systems in practice.

\subsection{Limitations and Future Research}

A first major limitation is our focus on only four PPC systems. More PPC systems, or different variants of the chosen PPC systems, could have been assessed. We focussed on these four 
systems since they are arguably the four most applied and well-known PPC systems for the production context under study, i.e. multi-stage assembly systems where the demand for components or product parts depends on the market-driven demand for end products. Future research could extend our study by also including other systems in the comparison, for example Constant Work-in-Process (ConWIP). Another important limitation is our choice of experimental factors, i.e. due date tightness and bottleneck severity. Although we consider these two factors the most likely to impact performance across PPC systems, future research could extend our study by including other environmental factors, such as processing time variability, machine failures, deterministic demand, bottleneck shiftiness etc. Finally, future research could also work on the integration of the different PPC systems. We observed significant overlap in the elements that constitute all four systems, with the main difference being in terms of which element is used, where it is used, and the degree of emphasis given to each element. We focused on well-established PPC systems whereas future research could deconstruct these systems into their underlying elements and assess the contingency factors for each element, thereby providing an architectural guide for PPC design.

\section{References}

Acosta, P.V.A., Mascle, C., \& Baptiste, P., 2020, Applicability of Demand-Driven MRP in a complex manufacturing environment, International Journal of Production Research, (in print).

Ardalan, A., \& Diaz, R., 2012, NERJIT: Using Net Requirement Data in Kanban-Controlled Jumbled-Flow Shops, Production \& Operations Management, 21, 3, 606-618.

Bokhorst, J.C.A., Slomp, J., \& Gaalman G.J.C., 2004, On the who-rule in Dual Resource Constrained (DRC) manufacturing systems, International Journal of Production Research, 42, 23, 5049-5074.

Chakravorty, S.S., \& Atwater, J.B., 2005, The impact of free goods on the performance of drum-buffer-rope scheduling systems, International Journal of Production Economics, 95, 347-357.

Craighead, C.W., Patterson, J.W., \& Fredendall, LD., 2001, Protective capacity positioning: impact on manufacturing cell performance, European Journal of Operational Research, 134, 425-438.

Enns, S.T., \& Prongue Costa, M., 2002, The effectiveness of input control based on aggregate versus bottleneck workloads, Production Planning \& Control, 13, 7, 614 - 624. 
Goldratt, E.M., 1990, What is This Thing Called Theory of Constraints and How Should it be Implemented?, North River Press: New York.

Goldratt, E.M. \& Cox, J., 1984, The Goal: Excellence in Manufacturing, North River Press: New York.

Golhar, D. Y., \& Stamm, C.L., 1991, The just-in-time philosophy: a literature review, International Journal of Production Research, 29, 4, 657-676.

Golmohammadi, D., 2015, A study of scheduling under the theory of constraints, International Journal of Production Economics, 165, 38-50.

Graves, S.C., 1986, A tactical planning model for a job shop, Operations Research, 34, 4, 522533.

Guide, V.D.R., \& Srivastava, R., 2000, A review of techniques for buffering against uncertainty with MRP systems, Production Planning \& Control, 11, 223-233.

Gupta, M., \& Snyder, D., 2009, Comparing TOC with MRP and JIT: a literature review, International Journal of Production Research, 47, 13, 3705-3739.

Ho, C.-J., 1989, Evaluating the impact of operating environments on MRP system nervousness, International Journal of Production Research, 27, 1115-1135.

Hopp, W., \& Spearman, M., 2011, Factory Physics, 3rd ed. Irwin: McGraw-Hill.

Jacobs, F.R., \& Weston Jr., F.C., 2007, Enterprise resource planning (ERP) - A brief history, Journal of Operations Management, 25, 357-363.

Ikeziri, L.M., Souza, F.B.D., Gupta, M.C. \& de Camargo Fiorini, P., 2019, Theory of constraints: review and bibliometric analysis, International Journal of Production Research, 57, 15-16, 5068-5102.

Jodlbauer, H. \& Huber, A., 2008, Service-level performance of MRP, kanban, CONWIP and DBR due to parameter stability and environmental robustness, International Journal of Production Research, 46, 8, 2179-2195.

Koh, S.C.L., Saad, S.M., \& Jones, M.H., 2002. Uncertainty under MRP-planned manufacture: Review and categorization, International Journal of Production Research, 40, 2399-2421.

Lage Junior, M. \& Godinho Filho, M., 2010, Variations of the kanban system: Literature review and classification, International Journal of Production Economics, 125, 13-21.

Lee, C-J., \& Rim S-C., 2019, A Mathematical Safety Stock Model for DDMRP Inventory Replenishment, Mathematical Problems in Engineering, Article ID 6496309.

Louly, M.A. \& Dolgui, A., 2013, Optimal MRP parameters for a single item inventory with random replenishment lead time, POQ policy and service level constraint, International Journal of Production Economics, 143, 1, 35-40. 
Mabin, V.J. \& Balderstone, S.J., 2003, The performance of the theory of constraints methodology: analysis and discussion of successful TOC applications, International Journal of Operations and Production Management, 23, 568-595.

Miclo, R. Lauras, M., Fontanili, F., Lamothe, J., \& Melnyk, S.A., 2019, Demand Driven MRP: assessment of a new approach to materials management, International Journal of Production Research, 57, 1, 166-181.

Missbauer, H., 2020, Order release planning by iterative simulation and linear programming: Theoretical foundation and analysis of its shortcomings, European Journal of Operational Research, 280, 2, 495-507.

Monden, Y., 1983, Toyota Production System: Practical Approach to Production Management, Industrial Engineering and Management Press, Norcross, Georgia.

Ohno, T., 1988, Toyota Production System: Beyond Large-Scale Production, $1^{\text {st }}$ Ed., Productivity Press.

Orlicky, J., 1975, Material Requirements Planning, New York, NY: McGraw-Hill.

Piplani, R. \& Ang, A.W.H., 2018, Performance comparison of multiple product kanban control systems, International Journal of Production Research, 56, 3, 1299-1312.

Ptak, C., \& Smith, C., 2011, Orlicky's Material Requirements Planning 3/E, New York: McGraw Hill Professional.

Ptak, C., \& Smith, C., 2016, Demand Driven Material Requirements Planning (DDMRP). Norwalk, CT: Industrial Press.

Radovilsky, Z.D., 1998, A quantitative approach to estimate the size of the time buffer in the theory of constraints, International Journal Production Economics, 55, 113-119.

Rahman, S., 1998, Theory of constraints: a review of the philosophy and its applications, International Journal of Operations \& Production Management, 18, 336-355.

Ronen B., \& Starr, M.K., 1990, Synchronized manufacturing as in OPT: from practice to theory, Computers \& Industrial Engineering, 18(4), 585-600.

Roser, C., \& Nakano, M., 2015, A quantitative comparison of bottleneck detection methods in manufacturing systems with particular consideration for shifting bottlenecks, IFIP International Conference on Advances in Production Management Systems (pp. 273-281).

Rossi, T., Pozzi, R., Pero, M. \& Cigolini, R., 2017, Improving production planning through finite-capacity MRP. International Journal of Production Research, 55, 2, 377-391.

Schragenheim, E. \& Ronen, B., 1990, Drum-buffer-rope shop floor control, Production and Inventory Management Journal, 31, 18-22. 
Simons, J.V. \& Simpson, III, W.P., 1997, An exposition of multiple constraint scheduling as implemented in the goal system (formerly disaster), Production \& Operations Management, $6,3-22$.

Steele, D.C., Philipoom, P.R., Malhotra, M.K., \& Fry T.D., 2005, Comparisons between drumbuffer-rope and material requirements planning: a case study, International Journal of Production Research, 43, 15, 3181-3208

Sugimori, Y., Kusunoki, K., Cho., F., \& Uchikawa, S., 1977, Toyota production system and Kanban system Materialization of just-in-time and respect-for-human system, International Journal of Production Research, 15, 6, 553-564.

Suri, R., 1998, Quick Response Manufacturing: A companywide approach to reducing leadtimes, Productivity Press.

Tenhiälä, A., 2011, Contingency theory of capacity planning: The link between process types and planning methods, Journal of Operations Management, 29, 1-2, 65-77.

Teo, C.C., Bhatnagar, R. \& Graves, S.C., 2012, An application of master schedule smoothing and planned lead time control, Production \& Operations Management, 21, 2, 211-223.

Teo, C.C., Bhatnagar, R. \& Graves, S.C., 2011, Setting planned lead times for a make-to-order production system with master schedule smoothing, IIE Transactions, 43, 6, 399-414.

Thürer, M., Fernandes, N.O. \& Stevenson, M., 2020, Material Flow Control in High-Variety Make-to-Order Shops: Combining COBACABANA and POLCA, Production \& Operations Management, (in print)

Thürer, M., Fernandes, N.O., Stevenson, M., Silva, C., \& Carmo-Silva, S., 2019; POLC-A: An Assessment of POLCA's Authorization Element, Journal of Intelligent Manufacturing, 30, $6,2435-2447$.

Thürer, M. \& Stevenson, M., 2018a, On the beat of the drum: improving the flow shop performance of the Drum-Buffer-Rope scheduling mechanism. International Journal of Production Research, 56, 9, 3294-3305.

Thürer, M., \& Stevenson, M., 2018b, Bottleneck-Oriented Order Release with Shifting Bottlenecks: An Assessment by Simulation, International Journal of Production Economics, 197, 275-282.

Thürer, M., Stevenson, M., \& Protzman, C.W., 2016, Card-Based Production Control: A Review of the Control Mechanisms Underpinning Kanban, ConWIP, POLCA and COBACABANA Systems, Production Planning \& Control, 27, 14, 1143-1157.

Thompson, J.D., 1967, Organizations in action: Social science bases for administrative theory, 1st Ed, Mc Graw-Hill book company, New York. 
Van Kampen, T.J., Van Donk, D.P. \& Van Der Zee, D.J., 2010, Safety stock or safety lead time: coping with unreliability in demand and supply, International Journal of Production Research, 48, 24, 7463-7481.

Vollmann, T.E., Berry, W.L., \& Whybark, D.C., 1997, Manufacturing Planning and Control Systems, Irwin: McGraw-Hill.

Watson, K.J., Blackstone, J.H., \& Gardiner, S.C., 2007, The evolution of a management philosophy: The theory of constraints, Journal of Operations Management, 25, 387-402.

White, R.E., \& Prybutok, V., 2001, The relationship between JIT practices and type of production system, Omega, 29, 113-124.

White, R.E., Pearson, J.N., \& Wilson, J.R., 1999, JIT manufacturing: A survey of implementation in small and large US manufacturers, Management Science, 45, 1, 1-15.

Wilson, J.M., 2016, The origin of material requirements planning in Frederick W. Taylor's planning office, International Journal of Production Research, 54, 5, 1535-1553.

Whybark, D.C., \& Williams, J.G., 1976, Material Requirements Planning under uncertainty, Decision Sciences, 7, 595-606. 
Table 1: Product Structure

\begin{tabular}{|c|c|c|c|c|c|c|c|c|c|c|c|c|c|c|c|}
\hline \multicolumn{3}{|c|}{ Station 1 (Final) } & \multicolumn{3}{|c|}{ Station 2} & \multicolumn{3}{|c|}{ Station 3} & \multicolumn{3}{|c|}{ Station 4} & \multicolumn{3}{|c|}{ Station 5} & \multirow{3}{*}{$\begin{array}{l}\text { Station } 6 \\
\text { Output }\end{array}$} \\
\hline \multirow{2}{*}{ Output } & \multicolumn{2}{|c|}{ Input } & \multirow{2}{*}{ Output } & \multicolumn{2}{|c|}{ Input } & \multirow{2}{*}{ Output } & \multicolumn{2}{|c|}{ Input } & \multirow{2}{*}{ Output } & \multicolumn{2}{|c|}{ Input } & \multirow{2}{*}{ Output } & \multicolumn{2}{|c|}{ Input } & \\
\hline & Type & $Q^{1)}$ & & Type & $\mathrm{Q}$ & & Type & $Q$ & & Type & $Q$ & & Type & $Q$ & \\
\hline Product 1 & B1 & 2 & B1 & C1 & 3 & C1 & D1 & 1 & D1 & E1 & 1 & E1 & $\mathrm{F} 1$ & 1 & F1 \\
\hline Product 2 & B2 & 2 & B2 & C1 & 1 & C1 & D1 & 1 & D1 & E1 & 1 & E1 & $\mathrm{F} 1$ & 1 & F1 \\
\hline Product 3 & B3 & 2 & B3 & C2 & 2 & C2 & D2 & 1 & D2 & E2 & 1 & E2 & F2 & 1 & F2 \\
\hline Product 4 & B4 & 2 & B4 & $\mathrm{C} 2$ & 2 & C2 & D2 & 1 & D2 & E2 & 1 & E2 & $\mathrm{F} 2$ & 1 & F2 \\
\hline Product 5 & B1 & 2 & B1 & C1 & 3 & C1 & D1 & 1 & D1 & E1 & 1 & E1 & $\mathrm{F} 1$ & 1 & F1 \\
\hline Product 6 & B2 & 2 & B2 & C1 & 1 & C1 & D1 & 1 & D1 & E1 & 1 & E1 & $\mathrm{F} 1$ & 1 & $\mathrm{~F} 1$ \\
\hline Product 7 & B3 & 2 & B3 & $\mathrm{C} 2$ & 2 & C2 & D2 & 1 & D2 & E2 & 1 & E2 & $\mathrm{F} 2$ & 1 & F2 \\
\hline Product 8 & B4 & 2 & B4 & C2 & 2 & C2 & D2 & 1 & D2 & E2 & 1 & E2 & F2 & 1 & F2 \\
\hline
\end{tabular}

Table 2: Simulation Results - Kanban

\begin{tabular}{|c|c|c|c|c|c|c|c|c|c|c|}
\hline \multirow{2}{*}{ Due Date } & \multirow{2}{*}{$\mathrm{K}^{1)}$} & \multicolumn{3}{|c|}{ No Bottleneck } & \multicolumn{3}{|c|}{ Moderate Bottleneck } & \multicolumn{3}{|c|}{ Severe Bottleneck } \\
\hline & & $\mathrm{SL}^{2)}(\%)$ & $\mathrm{FGI}^{3)}$ & $\mathrm{WIP}^{4)}$ & SL (\%) & FGI & WIP & SL (\%) & FGI & WIP \\
\hline Loose & 8 & 96.5 & 3.2 & 40.4 & 99.3 & 3.9 & 41.3 & 99.6 & 4.4 & 41.5 \\
\hline Medium & 8 & 95.7 & 3.2 & 40.4 & 99.0 & 3.9 & 41.3 & 99.5 & 4.4 & 41.5 \\
\hline Tight & 8 & 94.7 & 3.2 & 40.4 & 98.7 & 3.9 & 41.3 & 99.3 & 4.4 & 41.5 \\
\hline Loose & 16 & 99.6 & 9.3 & 84.6 & 99.9 & 10.4 & 84.3 & 100.0 & 11.0 & 83.7 \\
\hline Medium & 16 & 99.5 & 9.3 & 84.6 & 99.9 & 10.4 & 84.3 & 99.9 & 11.0 & 83.7 \\
\hline Tight & 16 & 99.4 & 9.3 & 84.6 & 99.9 & 10.4 & 84.3 & 99.9 & 11.0 & 83.7 \\
\hline Loose & 24 & 99.9 & 16.2 & 126.7 & 100.0 & 17.6 & 125.8 & 100.0 & 18.6 & 124.9 \\
\hline Medium & 24 & 99.9 & 16.2 & 126.7 & 100.0 & 17.6 & 125.8 & 100.0 & 18.6 & 124.9 \\
\hline Tight & 24 & 99.8 & 16.2 & 126.7 & 100.0 & 17.6 & 125.8 & 100.0 & 18.6 & 124.9 \\
\hline
\end{tabular}


Table 3: Simulation Results - MRP

\begin{tabular}{|c|c|c|c|c|c|c|c|c|c|c|c|}
\hline \multirow{2}{*}{ Due Date } & \multirow{2}{*}{$\mathrm{TB}^{1)}$} & \multirow{2}{*}{$\mathrm{IB}^{2)}$} & \multicolumn{3}{|c|}{ No Bottleneck } & \multicolumn{3}{|c|}{ Moderate Bottleneck } & \multicolumn{3}{|c|}{ Severe Bottleneck } \\
\hline & & & $\mathrm{SL}^{3)}(\%)$ & $F G I^{4)}$ & WIP5) & SL (\%) & $\mathrm{FGI}$ & WIP & SL (\%) & $\mathrm{FGI}$ & WIP \\
\hline Loose & 0 & 0 & 20.5 & 0.4 & 48.3 & 18.9 & 0.3 & 38.1 & 18.5 & 0.2 & 32.5 \\
\hline Medium & 0 & 0 & 20.4 & 0.4 & 47.2 & 19.0 & 0.3 & 38.4 & 18.6 & 0.2 & 32.7 \\
\hline Tight & 0 & 0 & 19.1 & 0.4 & 44.0 & 18.7 & 0.3 & 37.7 & 18.7 & 0.2 & 32.7 \\
\hline Loose & 0 & 8 & 77.6 & 7.9 & 86.5 & 81.6 & 8.0 & 83.1 & 83.7 & 8.0 & 77.3 \\
\hline Medium & 0 & 8 & 78.1 & 8.1 & 81.8 & 81.9 & 8.0 & 81.2 & 83.5 & 7.9 & 77.5 \\
\hline Tight & 0 & 8 & 77.8 & 8.0 & 77.3 & 81.7 & 8.0 & 76.9 & 83.7 & 8.0 & 76.4 \\
\hline Loose & 0 & 16 & 92.2 & 16.1 & 125.4 & 95.0 & 16.1 & 123.8 & 96.1 & 16.0 & 118.2 \\
\hline Medium & 0 & 16 & 92.3 & 16.0 & 120.9 & 94.9 & 16.1 & 120.6 & 96.0 & 16.0 & 118.3 \\
\hline Tight & 0 & 16 & 92.2 & 16.0 & 116.5 & 94.9 & 16.0 & 116.2 & 96.1 & 16.0 & 116.0 \\
\hline Loose & 2 & 0 & 30.9 & 0.9 & 48.4 & 33.0 & 0.8 & 38.1 & 35.2 & 0.7 & 32.9 \\
\hline Medium & 2 & 0 & 30.4 & 0.9 & 46.0 & 32.8 & 0.8 & 38.4 & 34.8 & 0.7 & 32.6 \\
\hline Tight & 2 & 0 & 28.7 & 0.8 & 42.6 & 33.0 & 0.8 & 38.1 & 34.4 & 0.7 & 32.6 \\
\hline Loose & 2 & 8 & 83.4 & 9.5 & 84.6 & 88.0 & 9.5 & 82.7 & 90.2 & 9.5 & 77.6 \\
\hline Medium & 2 & 8 & 83.6 & 9.4 & 80.2 & 88.2 & 9.5 & 79.4 & 90.4 & 9.5 & 77.4 \\
\hline Tight & 2 & 8 & 83.4 & 9.4 & 75.6 & 88.0 & 9.5 & 82.7 & 90.4 & 9.5 & 74.8 \\
\hline Loose & 2 & 16 & 94.3 & 17.7 & 123.7 & 96.9 & 17.8 & 122.9 & 98.0 & 17.8 & 118.5 \\
\hline Medium & 2 & 16 & 94.3 & 17.8 & 119.0 & 96.8 & 17.7 & 118.9 & 97.9 & 17.8 & 118.1 \\
\hline Tight & 2 & 16 & 94.5 & 17.9 & 114.5 & 96.9 & 17.8 & 114.3 & 98.0 & 17.8 & 114.3 \\
\hline Loose & 4 & 0 & 40.9 & 1.5 & 48.0 & 46.0 & 1.5 & 38.0 & 49.9 & 1.5 & 32.6 \\
\hline Medium & 4 & 0 & 39.4 & 1.5 & 44.9 & 45.9 & 1.5 & 38.0 & 50.2 & 1.5 & 32.7 \\
\hline Tight & 4 & 0 & 37.0 & 1.3 & 41.6 & 45.9 & 1.5 & 37.4 & 50.5 & 1.5 & 32.4 \\
\hline Loose & 4 & 8 & 87.6 & 11.0 & 82.9 & 92.4 & 11.1 & 81.7 & 94.6 & 11.2 & 77.5 \\
\hline Medium & 4 & 8 & 87.8 & 11.0 & 78.3 & 92.5 & 11.1 & 77.7 & 94.7 & 11.2 & 76.8 \\
\hline Tight & 4 & 8 & 87.4 & 10.9 & 73.9 & 92.3 & 11.1 & 73.2 & 94.5 & 11.2 & 73.0 \\
\hline Loose & 4 & 16 & 96.0 & 19.5 & 121.8 & 98.0 & 19.5 & 121.4 & 98.9 & 19.5 & 118.5 \\
\hline Medium & 4 & 16 & 95.8 & 19.4 & 117.3 & 98.1 & 19.5 & 117.1 & 98.9 & 19.6 & 116.8 \\
\hline Tight & 4 & 16 & 95.9 & 19.4 & 112.8 & 98.1 & 19.5 & 112.6 & 98.9 & 19.5 & 112.5 \\
\hline
\end{tabular}


Table 4: Simulation Results - OPT (DBR)

\begin{tabular}{|c|c|c|c|c|c|c|c|c|c|c|c|}
\hline \multirow{2}{*}{ Due Date } & \multirow{2}{*}{$\mathrm{TB}^{1)}$} & \multirow{2}{*}{$\mathrm{IB}^{2)}$} & \multicolumn{3}{|c|}{ No Bottleneck } & \multicolumn{3}{|c|}{ Moderate Bottleneck } & \multicolumn{3}{|c|}{ Severe Bottleneck } \\
\hline & & & $\mathrm{SL}^{3)}(\%)$ & $\left.F G\right|^{4)}$ & WIP5) & SL (\%) & FGI & WIP & SL (\%) & FGI & WIP \\
\hline Loose & 0 & 16 & 28.6 & 3.2 & 23.1 & 65.3 & 9.5 & 21.4 & 98.5 & 8.9 & 28.2 \\
\hline Medium & 0 & 16 & 30.4 & 3.5 & 23.1 & 66.1 & 9.6 & 21.3 & 98.0 & 8.9 & 28.2 \\
\hline Tight & 0 & 16 & 28.9 & 3.3 & 23.1 & 65.5 & 9.5 & 21.4 & 97.3 & 8.9 & 28.2 \\
\hline Loose & 0 & 24 & 79.4 & 17.3 & 32.0 & 91.0 & 21.0 & 29.9 & 99.6 & 12.1 & 33.7 \\
\hline Medium & 0 & 24 & 79.4 & 17.6 & 32.2 & 90.6 & 20.9 & 29.9 & 99.4 & 12.1 & 33.7 \\
\hline Tight & 0 & 24 & 79.7 & 17.3 & 32.3 & 91.2 & 21.2 & 29.9 & 99.2 & 12.1 & 33.7 \\
\hline Loose & 0 & 32 & 93.4 & 28.6 & 40.7 & 96.7 & 30.6 & 38.0 & 99.7 & 12.3 & 35.7 \\
\hline Medium & 0 & 32 & 93.0 & 28.4 & 40.8 & 96.8 & 30.8 & 38.0 & 99.5 & 12.3 & 35.7 \\
\hline Tight & 0 & 32 & 93.0 & 28.5 & 40.6 & 96.5 & 30.7 & 38.1 & 99.3 & 12.3 & 35.7 \\
\hline Loose & 2 & 16 & 34.8 & 4.4 & 23.1 & 69.6 & 10.9 & 21.4 & 99.2 & 11.6 & 35.4 \\
\hline Medium & 2 & 16 & 35.0 & 4.4 & 23.0 & 69.4 & 10.7 & 21.4 & 98.9 & 11.6 & 35.4 \\
\hline Tight & 2 & 16 & 32.2 & 3.9 & 22.9 & 70.4 & 10.9 & 21.4 & 98.5 & 11.6 & 35.4 \\
\hline Loose & 2 & 24 & 80.4 & 18.1 & 32.2 & 92.9 & 22.9 & 29.9 & 99.8 & 15.6 & 39.9 \\
\hline Medium & 2 & 24 & 80.8 & 18.4 & 32.2 & 92.9 & 22.8 & 29.9 & 99.7 & 15.6 & 39.9 \\
\hline Tight & 2 & 24 & 81.9 & 18.5 & 32.2 & 92.0 & 22.5 & 29.9 & 99.5 & 15.6 & 39.9 \\
\hline Loose & 2 & 32 & 94.6 & 30.2 & 40.7 & 97.2 & 32.4 & 38.1 & 99.9 & 19.1 & 43.2 \\
\hline Medium & 2 & 32 & 94.4 & 30.3 & 40.7 & 97.4 & 32.5 & 38.1 & 99.9 & 19.1 & 43.2 \\
\hline Tight & 2 & 32 & 94.8 & 30.3 & 40.6 & 97.3 & 32.3 & 38.1 & 99.8 & 19.1 & 43.2 \\
\hline Loose & 4 & 16 & 37.2 & 4.8 & 23.1 & 74.1 & 12.2 & 21.4 & 99.5 & 14.4 & 42.1 \\
\hline Medium & 4 & 16 & 34.4 & 4.5 & 23.0 & 73.9 & 12.2 & 21.4 & 99.3 & 14.4 & 42.1 \\
\hline Tight & 4 & 16 & 36.8 & 5.0 & 23.1 & 73.3 & 12.1 & 21.4 & 99.0 & 14.4 & 42.1 \\
\hline Loose & 4 & 24 & 82.8 & 20.1 & 32.2 & 94.1 & 24.3 & 29.9 & 99.6 & 18.8 & 44.6 \\
\hline Medium & 4 & 24 & 84.2 & 20.1 & 32.0 & 94.1 & 24.3 & 29.9 & 99.5 & 18.8 & 44.6 \\
\hline Tight & 4 & 24 & 83.4 & 20.0 & 32.2 & 93.6 & 24.3 & 29.9 & 99.3 & 18.8 & 44.6 \\
\hline Loose & 4 & 32 & 95.3 & 31.9 & 40.6 & 98.0 & 34.0 & 38.1 & 99.9 & 23.0 & 48.8 \\
\hline Medium & 4 & 32 & 95.4 & 32.1 & 40.7 & 98.0 & 34.1 & 38.1 & 99.8 & 23.0 & 48.8 \\
\hline Tight & 4 & 32 & 95.0 & 31.6 & 40.7 & 98.2 & 34.4 & 38.1 & 99.7 & 23.0 & 48.8 \\
\hline
\end{tabular}


Table 5: Simulation Results - DDMRP

\begin{tabular}{|c|c|c|c|c|c|c|c|c|c|c|c|}
\hline \multirow{2}{*}{ Due Date } & \multirow{2}{*}{$\mathrm{ROP}^{1)}$} & \multirow{2}{*}{$\mathrm{IB}^{2)}$} & \multicolumn{3}{|c|}{ No Bottleneck } & \multicolumn{3}{|c|}{ Moderate Bottleneck } & \multicolumn{3}{|c|}{ Severe Bottleneck } \\
\hline & & & $\mathrm{SL}^{3)}(\%)$ & $\mathrm{FGI}^{4)}$ & WIP5) & SL (\%) & FGI & WIP & SL (\%) & FGI & WIP \\
\hline Loose & 1 & 24 & 77.8 & 4.2 & 28.3 & 95.3 & 7.3 & 28.3 & 98.5 & 8.9 & 28.2 \\
\hline Medium & 1 & 24 & 75.6 & 4.2 & 28.3 & 94.2 & 7.3 & 28.3 & 98.0 & 8.9 & 28.2 \\
\hline Tight & 1 & 24 & 73.1 & 4.2 & 28.3 & 92.9 & 7.3 & 28.3 & 97.3 & 8.9 & 28.2 \\
\hline Loose & 2 & 24 & 96.7 & 8.7 & 35.3 & 99.2 & 11.0 & 34.3 & 99.6 & 12.1 & 33.7 \\
\hline Medium & 2 & 24 & 95.9 & 8.7 & 35.3 & 99.0 & 11.0 & 34.3 & 99.4 & 12.1 & 33.7 \\
\hline Tight & 2 & 24 & 94.9 & 8.7 & 35.3 & 98.6 & 11.0 & 34.3 & 99.2 & 12.1 & 33.7 \\
\hline Loose & 3 & 24 & 97.2 & 9.1 & 37.8 & 99.4 & 11.1 & 36.7 & 99.7 & 12.3 & 35.7 \\
\hline Medium & 3 & 24 & 96.5 & 9.1 & 37.8 & 99.2 & 11.1 & 36.7 & 99.5 & 12.3 & 35.7 \\
\hline Tight & 3 & 24 & 95.7 & 9.1 & 37.8 & 98.9 & 11.1 & 36.7 & 99.3 & 12.3 & 35.7 \\
\hline Loose & 1 & 32 & 88.5 & 7.0 & 36.3 & 97.6 & 10.2 & 35.7 & 99.2 & 11.6 & 35.4 \\
\hline Medium & 1 & 32 & 86.9 & 7.0 & 36.3 & 97.0 & 10.2 & 35.7 & 98.9 & 11.6 & 35.4 \\
\hline Tight & 1 & 32 & 85.0 & 7.0 & 36.3 & 96.2 & 10.2 & 35.7 & 98.5 & 11.6 & 35.4 \\
\hline Loose & 2 & 32 & 98.2 & 11.9 & 41.7 & 99.6 & 14.3 & 40.6 & 99.8 & 15.6 & 39.9 \\
\hline Medium & 2 & 32 & 97.7 & 11.9 & 41.7 & 99.5 & 14.3 & 40.6 & 99.7 & 15.6 & 39.9 \\
\hline Tight & 2 & 32 & 97.1 & 11.9 & 41.7 & 99.3 & 14.3 & 40.6 & 99.5 & 15.6 & 39.9 \\
\hline Loose & 3 & 32 & 99.1 & 15.5 & 45.6 & 99.8 & 17.6 & 44.5 & 99.9 & 19.1 & 43.2 \\
\hline Medium & 3 & 32 & 98.8 & 15.5 & 45.6 & 99.6 & 17.6 & 44.5 & 99.9 & 19.1 & 43.2 \\
\hline Tight & 3 & 32 & 98.4 & 15.5 & 45.6 & 99.5 & 17.6 & 44.5 & 99.8 & 19.1 & 43.2 \\
\hline Loose & 1 & 40 & 92.3 & 9.4 & 44.0 & 98.4 & 12.6 & 43.0 & 99.5 & 14.4 & 42.1 \\
\hline Medium & 1 & 40 & 91.0 & 9.4 & 44.0 & 98.0 & 12.6 & 43.0 & 99.3 & 14.4 & 42.1 \\
\hline Tight & 1 & 40 & 89.5 & 9.4 & 44.0 & 97.4 & 12.6 & 43.0 & 99.0 & 14.4 & 42.1 \\
\hline Loose & 2 & 40 & 96.8 & 14.4 & 45.2 & 99.3 & 17.4 & 45.0 & 99.6 & 18.8 & 44.6 \\
\hline Medium & 2 & 40 & 96.2 & 14.4 & 45.2 & 99.1 & 17.4 & 45.0 & 99.5 & 18.8 & 44.6 \\
\hline Tight & 2 & 40 & 95.4 & 14.4 & 45.2 & 98.8 & 17.4 & 45.0 & 99.3 & 18.8 & 44.6 \\
\hline Loose & 3 & 40 & 99.1 & 19.0 & 51.2 & 99.8 & 21.5 & 49.9 & 99.9 & 23.0 & 48.8 \\
\hline Medium & 3 & 40 & 98.9 & 19.0 & 51.2 & 99.7 & 21.5 & 49.9 & 99.8 & 23.0 & 48.8 \\
\hline Tight & 3 & 40 & 98.6 & 19.0 & 51.2 & 99.6 & 21.5 & 49.9 & 99.7 & 23.0 & 48.8 \\
\hline
\end{tabular}


Table 6: Summary of Coordination Mechanisms Underpinning Each PPC System

\begin{tabular}{rcc}
\hline & $\begin{array}{c}\text { Coordination by mutual adjustment } \\
\text { (replenishment trigger) }\end{array}$ & $\begin{array}{c}\text { Coordination by plan } \\
\text { (backward scheduling) }\end{array}$ \\
\hline Kanban & All parts & None \\
MRP & None & All parts \\
OPT & Parts produced by non-constraints & Parts produced by constraints \\
DDMRP & Critical parts & Non-critical parts \\
\hline
\end{tabular}

Table 7: Summary of Applicability of PPC Systems

\begin{tabular}{|r|l|}
\hline Contingency Factor & Impact on PPC system performance \\
\hline $\begin{array}{r}\text { Demand Driven High } \\
\text { Variability }\end{array}$ & $\begin{array}{l}\text { Need for some form of mutual adjustment. Kanban, OPT and DDMRP perform } \\
\text { the best. For MRP, the production start date should not be enforced but used to } \\
\text { guide dispatching decisions. }\end{array}$ \\
\hline Due Date Tightness & $\begin{array}{l}\text { Has a negligible effect on realized operation throughput times. This means that } \\
\text { the impact on the service level can be calculated. }\end{array}$ \\
\hline Bottleneck Severity & $\begin{array}{l}\text { Kanban and DDMRP perform the best if there is no bottleneck. If there is a } \\
\text { bottleneck, then DDMRP and OPT perform the best, with DDMRP realizing lower } \\
\text { inventory levels. If there is a severe bottleneck, then the performance of DDMRP } \\
\text { and OPT converges. If there is a shifting bottleneck, then Kanban should be } \\
\text { chosen since both DDMRP and OPT rely on the bottleneck being known. }\end{array}$ \\
\hline
\end{tabular}

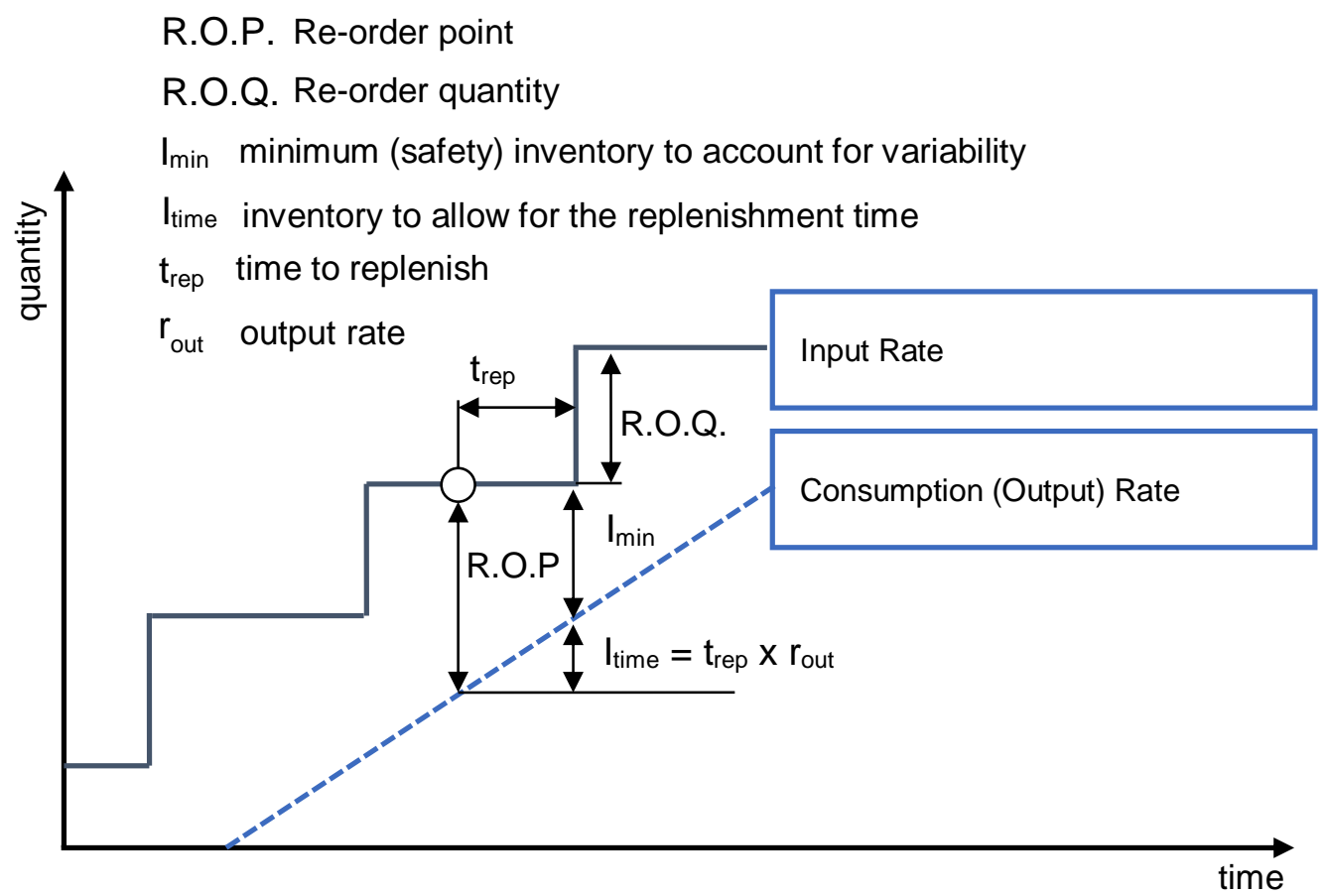

Figure 1: Illustration of a Re-Order System 


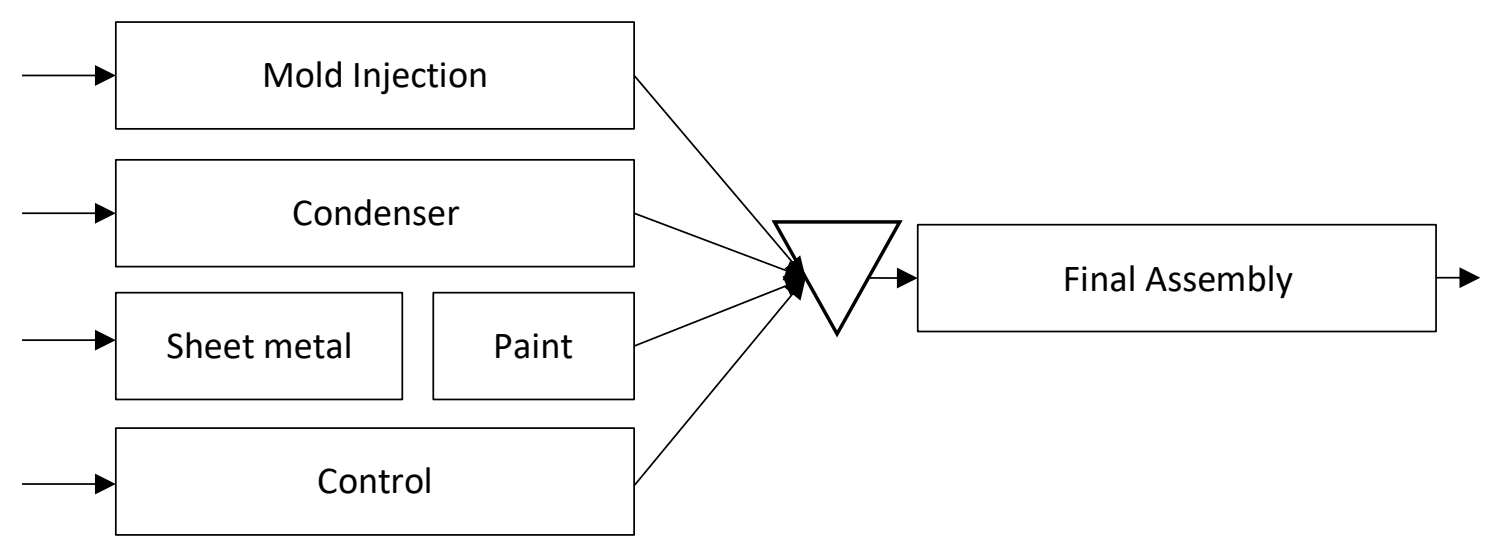

Figure 2: Illustration of Production Process at Triggering Case Company 